Collaboration in the Last Mile: Evidence from Grocery Deliveries

Emel Aktas ${ }^{1}$, Michael Bourlakis ${ }^{1}$, Dimitris Zissis ${ }^{2}$

${ }^{1}$ Cranfield School of Management, Cranfield University, Cranfield, UK

${ }^{2}$ Norwich Business School, University of East Anglia, Norwich, UK

Corresponding Author: Emel Aktas (emel.aktas@cranfield.ac.uk) 


\title{
Collaboration in the Last Mile: Evidence from Grocery Deliveries
}

\begin{abstract}
The grocery sector has transitioned into an omnichannel operating mode, allowing consumers to buy online and have their order delivered to their chosen address. The last mile delivery service leads to avoidable inefficiencies such as low asset utilisation and repeated trips to nearby neighbourhoods, increasing vehicle emissions, traffic, and operational costs. Combining historical order and delivery data of an online grocery retailer with secondary data publicly available on other retailers, we employ Monte Carlo simulation to estimate grocery home delivery demand per 1-hour time windows. We use the simulation output as an input to daily vehicle routing problem instances under independent and collaborative last mile delivery operation to estimate the impact of collaboration. Our analyses show distance savings of around $17 \%$ and route reduction of around $22 \%$. These results can support policies incentivising vehicle and infrastructure sharing settings and decoupling the last mile delivery from the core grocery retail services.
\end{abstract}

Keywords: Collaboration; last mile delivery; UK grocery sector; e-commerce; vehicle routing; Monte Carlo simulation

\section{Introduction}

Managing urban areas has become one of the most significant development challenges of the $21^{\text {st }}$ century as the urban population has grown from 746 million in 1950 to 7.6 billion in 2019 (https://www.census.gov/popclock/). Considering that the world's population in 2050 is projected to be $66 \%$ urban (UN, 2015) with 41 mega-cities having more than 10 million inhabitants by 2030 , urgent attention on urban planning is required for easy access to education, healthcare, infrastructure, and services. Transport constitutes a key aspect of the smooth functioning city life and urban freight transport has a significant negative impact on the quality of life through traffic congestion, vehicle emissions, and noise pollution (Nathanail et al., 2017). 
Increased urbanisation has led to an increased demand for not only food products but also all goods and services to be made available in urban areas. The developments in information and communication technologies and the Internet have enabled new convenience services such as online shopping using desktop computers and more recently mobile devices to access and purchase books, apparel, flight tickets, or hotel bookings. As mobile devices get smarter and faster, online ordering becomes a ubiquitous aspect of any consumer-facing industry.

The food sector has also benefited from increasing e-commerce. In addition to the rapidly emerging new online delivery concepts such as meal deliveries or fresh produce boxes, retailers in many countries have also invested in infrastructure and distribution networks to make grocery purchase an online service available to their consumers at their convenience. Especially cash-rich, time-poor city dwellers have demonstrated a growing preference for shopping their groceries online and demanding their orders to be delivered to their homes on their preferred day and time.

The UK online grocery sector has seen a growth of $13.5 \%$ in 2017 reaching a market size of $£ 11.31$ billion (Mintel, 2018). Major retailers such as Tesco, Sainsbury’s, Ocado, and Asda operate their own vehicle fleets to fulfil their home delivery demand. Classified under the last mile logistics, home delivery is the most expensive segment of the transportation process with the lowest efficiency compared to other activities in the supply chain (Lim et al., 2018). Due to the nature of the service, multiple and uncoordinated vehicles visit the same neighbourhood at around the same time, increasing the last mile distribution costs as well as the negative impact of the operation on the environment.

Online grocery purchase and delivery services are recognised as a key offering by major retailers in the UK. Unfortunately, the fierce competition and the constant 
requirement to 'delight the customers' has resulted in retailers' investing in their own fleets to satisfy the home delivery demand with inevitable inefficiencies in the distribution operation. In fact, in line with the growing e-commerce and delivery services, the RAC Foundation estimates the light commercial vehicles to double in number by 2035, compared to 2003 figures (Clarke et al., 2014). The increasing number of delivery vans also poses a societal challenge in terms of increasing traffic congestion and noise pollution as well as increasing likelihood of road accidents. As cities get more crowded, consolidation and sharing in delivery services will become inevitable, an issue investigated in this paper which needs to be considered carefully by retailers. Likewise, further work is already under way to transition into cloud services for grocery shopping and delivery by enabling Internet of Things sensors and delivery robots pending developing the necessary security and privacy mechanisms (Al Sunny et al., 2019).

On this basis, the aim of this paper is to investigate efficiency gains from shared logistics in the last mile distribution under plausible collaborative logistics scenarios to be followed by UK grocery retailers, the "problem owners" in this work. For this purpose, we first conceptualise a micro hub located near residential areas with a short delivery radius for the last mile deliveries of groceries. Then, we use the capacitated vehicle routing problem with time windows (CVRPTW) to model the online-purchased grocery last mile distribution. In the vehicle routing problem (VRP), the demands of customers are fulfilled with the products originating from a depot and transported using a fleet of vehicles such that the total traveling cost of all vehicles is minimised (Abdulkader et al., 2018). It imposes capacity constraints that are smaller than the total demand of all locations to be visited with multiple vehicles meeting the total demand in the service area. Such multiple vehicles, represented as the number of routes in the output of the model, are a good estimator of the fleet size. 
The major contributions of this paper can be summarised as follows. We investigate a problem that is gaining exponential importance: home deliveries by UK grocery retailers for food products purchased online. Due to the time-sensitive nature of grocery home deliveries, we develop an integrated method for estimating the grocery demand and fulfilling home deliveries using vehicle routing problem with time windows. Based on primary delivery data from an online retailer and aggregate secondary data on other online retailers from published reports, we estimate grocery home delivery demand in a postcode sector in London, where retailers compete fiercely to maintain and increase their online grocery market share. Simulating home delivery demand over a year for two hypothetical retailers informed by real data, we estimate distance and route gains from logistics sharing in the last mile delivery. We adopt the concept of micro hubs which enable logistics asset sharing for the last mile of the grocery delivery for UK retailers and report potential benefits from collaboration whilst adhering to the realities of grocery home deliveries such as up to 10 and 15 drops per route and one-hour time windows for deliveries.

The remainder of the paper is organised as follows: Section 2 provides the literature review. Section 3 defines the methodology adopted for measuring benefits when online retailers collaborate in the last mile delivery services. Section 4 presents the findings and the discussion is provided in Section 5. Finally, Section 6 highlights the conclusions and sets the questions for future research.

\section{Literature review}

The UK grocery retail sector is known for its severe competition (Hackney et al., 2006) and the sustainability of distribution operation is yet to be established for home deliveries of groceries purchased online. This is mainly due to the high impact of the online channel on the physical network that fulfils the service demand together with 
stringent service parameters such as 1-hour delivery windows and booking of deliveries in advance. Shrinking profit margins for e-commerce coupled with consumers' increasing service expectations on the timeliness of deliveries necessitate careful planning of small shipments (Cleophas \& Ehmke, 2014).

Like other online retail services, online grocery purchase and the subsequent home delivery service change consumers' shopping habits. Convenience comes at an economic, environmental, and social cost in the form of higher prices, increasing carbon emissions, and additional congestion on the roads. The online way of shopping groceries affects grocery retail revenue models as well as carbon emissions in the last mile distribution, due to increased convenience through two dominant models in the market: pay-per-order and subscription-based delivery service (Belavina et al., 2017). A comparison of financial and environmental performance of these models suggests subscription-based service increases emissions from deliveries whilst reducing the food waste whereas pay-per-order service is preferable by retailers, especially in sparsely populated geographies with high delivery costs.

Numerous factors such as drop density, distance, and vehicle type affect the emissions from home delivery services. Emissions from the average shopping trip of a consumer, particularly by private car, can be greater than the emissions from all upstream logistics activities (Edwards et al., 2010). On the other hand, emissions from delivery vans can be reduced if it is possible to combine the deliveries over spatially and temporally comparable grocery orders. In that respect, a classic combinatorial optimisation problem, vehicle routing, has become a key aspect of managing distribution operations.

In fact, the retailer's physical network characterised by the density, size, and location of stores affects not only operating costs but also environmental costs (Cachon, 
2014). It has been a long debate whether consumers' travelling to stores causes higher carbon emissions than retailers' delivering orders to consumers' homes. The answer is not straightforward as it is affected by not only the store network, but also the shopping preferences and the shopping frequency of consumers. The store network could comprise few and far stores where the journey to the store takes a significant travel distance and time or many and close stores where the shopping trips are shorter. Consumers may perceive shopping as a leisure time activity and allocate several hours of travel and shopping time on a regular basis or as a chore that has to be done quickly and at minimum cost.

Shared capacity routing for omni-channel where customers order goods online and then pick them up in a store can lead to substantial savings by sharing vehicle capacity across different channels (Paul et al., 2019). With a capacity sharing strategy, the retailer exploits the spare capacity in its transport operations and reduces the transport costs as well as the number of customer visits. An integrated distribution system within the retailer's own network is found to reduce the distribution cost by up to $44 \%$ through sharing vehicles between physical and online channel (Abdulkader et al., 2018).

To address the demand for grocery delivery, retailers have developed their own logistics operations and have avoided logistics sharing with other retailers despite possible benefits such as cost savings from consolidating freight (Lozano et al., 2013). There is also the case of the Austrian grocery industry in which parties are able to improve their profitability by sharing information and setting up business with valueadding partnerships; even if these are competitors, proving that competition and collaboration can occur at the same time (Kotzab \& Teller, 2003). 
Although the literature suggests that online grocery retail firms do not actively engage in anticipating, experimenting, or determining which consumer expectations might result in a competitive advantage (de Kervenoael et al., 2016), the evidence from the UK is opposite: online grocery retailers in the UK consider the logistics service an integral element of their value-added service and provide it using their own fleets, at times with low utilisation. This is the gap we are addressing in this paper by showing theoretical gains from logistics sharing whilst retailers continue to compete in other core parts of the business.

Collaboration among supply chain actors, especially in the food distribution can not only increase load factors but also improve flexibility to accommodate peaks in demand (Rogerson \& Santén, 2017). Especially the retailers have a strong position to create conditions for more efficient supply chains and balanced delivery networks, improving the sustainability of the logistics operation (Accorsi et al., 2018). In line with this, shared capacity routing problem provides feasible solutions to retailers to manage their omni-channel distribution operation (Paul et al., 2019). To the best of the authors' knowledge, this work is one of the first research papers on capacity sharing in the last mile logistics of grocery deliveries to consumers using primary data, in a rapidly growing research stream of online delivery problem.

\section{Methodology}

Following on from the theoretical basis explained above, we propose a shared facility, which we will refer to as a micro hub, to be located near residential areas with a service range of 2-km radius to be able to serve postcode sectors in London with an average area of $1.40 \mathrm{~km}^{2}$ and a standard deviation of $1.13 \mathrm{~km}^{2}$. The micro hub will serve as a cross-docking facility for the last mile distribution. Large flows from picking locations of retailers are transported to micro hubs in residential areas, and then the last mile 
distribution is performed from the micro hub in line with promised time windows. We present the conceptualisation of the online grocery distribution in Figure 1 with two shared logistics models:

(1) Shared logistics in the stem mile: large flows from retailer's picking locations to the micro hubs.

(2) Shared logistics in the last mile: small flows from the micro hubs to the consumer's preferred locations.

The flows we consider for analyses in this paper are based on the last mile, ie small flows from micro hubs to consumers' addresses with time window constraints. We refer the readers to Zissis et al. (2018) for the shared logistics in the stem mile. This paper complements earlier work in its focus on the small flows.

\begin{tabular}{|c|c|c|c|c}
$\begin{array}{c}\text { Locations of } \\
\text { Retailers }\end{array}$ & Large flows & $\begin{array}{c}\text { Micro Hub in } \\
\text { Postcode } \\
\text { Sector }\end{array}$ & Small flows & $\begin{array}{c}\text { Consumers' } \\
\text { Addresses }\end{array}$ \\
\cline { 3 - 4 }
\end{tabular}

Figure 1 Main logistics flows in home deliveries of groceries

In modelling the grocery last mile distribution, we have an integrated methodology (Figure 2) to test the potential benefits of shared logistics: demand estimation and CVRPTW for small flows. In the demand estimation stage, we estimate the annual grocery orders to be delivered in postcode sectors of London using three data sources: 1) online grocery orders collected from annual reports of retailers including average basket size which we used to estimate how many orders each retailer has per year based on their total annual sales, 2) retailer's geographical store footprint, which comprises the number of stores and the size of those stores in the study area, obtained by the researchers via a web scaping exercise, and 3) population and socioeconomic data retrieved from the UK Office of National Statistics. 
A UK-grocery retailer has provided primary data on its home delivery services of groceries purchased online. The data set contains 346,745 orders from 533 postcode sectors in London from 1st June 2014 to 31 st May 2015. First, we ran Monte Carlo simulation to distribute the annual orders across the days of the year, using proportional distribution of orders across the days of the year from the primary data provider. Additionally, we use results from an online consumer survey (Zissis, Aktas, et al., 2018) for their time preferences to receive grocery orders in Monte Carlo simulations to distribute daily orders across 1-hour time windows throughout the day. We then take this demand as input to the CVRPTW and solve the daily grocery last mile delivery problem with one-hour time windows minimising the total distance travelled in the objective function. Using a combination of primary and secondary data we investigate the impact of vehicle capacity (two capacities tested) and logistics operation (independent and shared) between two retailers. 


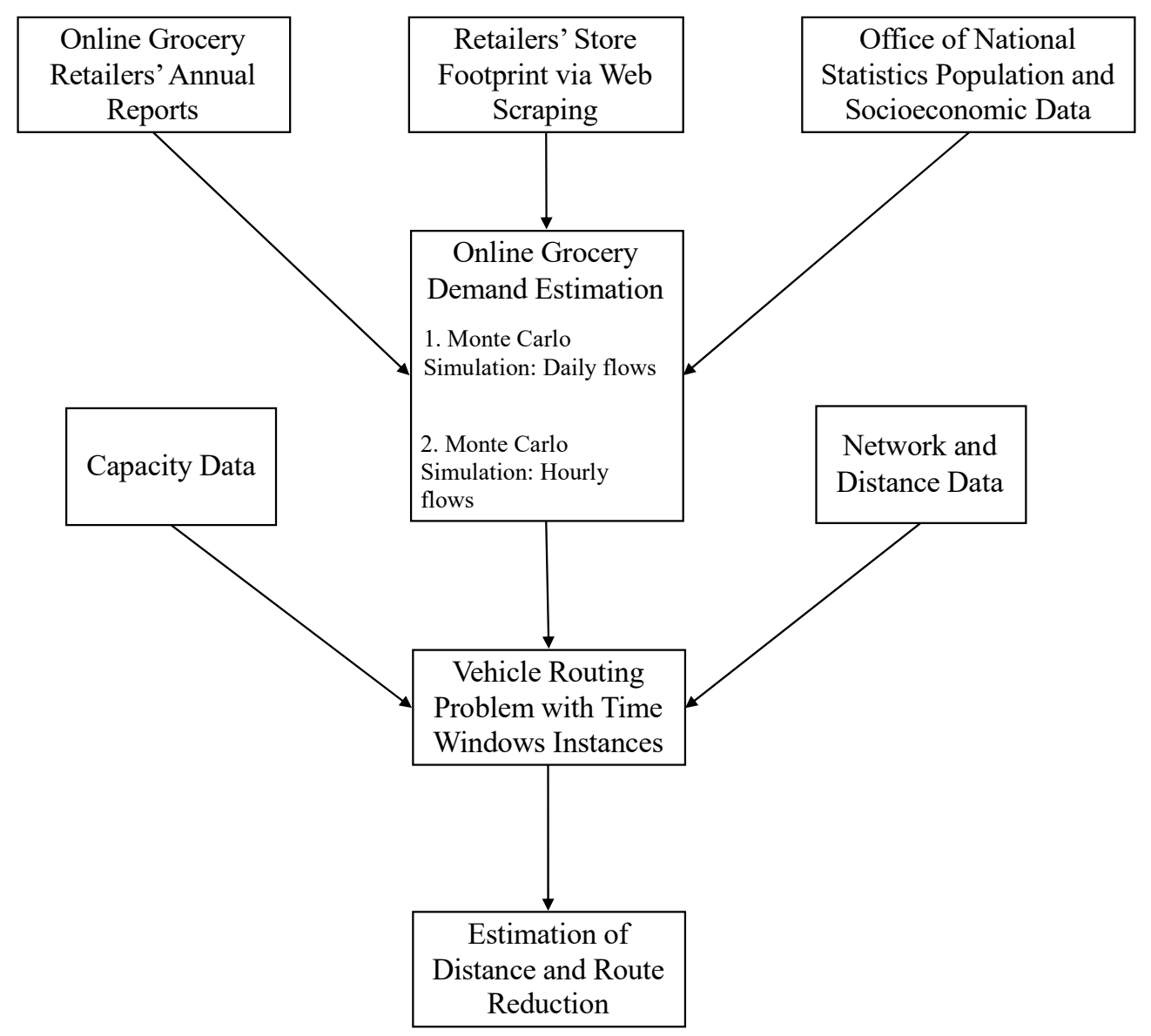

Figure 2 Methodology for estimating the effect of collaboration on distance and route

As customers choose what time they would like to receive their groceries, we use the CVRPTW for the grocery last mile deliveries from micro hubs to consumers' preferred locations, incorporating the delivery time window. The CVRPTW is formulated (Cordeau et al., 2007) as follows. Vertices 0 and $n+1$ denote the micro hub. Customers (in this problem, consumers) are denoted with the set $N=\{1,2, \ldots, n\}$. $V$ is the set of vertices, $V=\{0,1,2, \ldots, n, n+1\}$ where the first $(0)$ and the last elements $(n+1)$ correspond to the micro hub. $K$ is the set of vehicles, each with the same capacity. Each customer $i$ must be served within the time windows $\left[a_{i}, b_{i}\right]$ where $a_{i}$ is the start and $b_{i}$ is the end of the time window. The service time for each customer is $s_{i}$ and $w_{i k}$ indicates the time at which vehicle $k$ starts servicing customer $i$. The binary decision variables $x_{i j k}$ take the value 1 if the vehicle $k$ travels from vertex $i$ to 
vertex $j$, otherwise zero. The time it takes to travel from vertex $i$ to vertex $j$ is denoted as $t_{i j}$ while $c_{i j}$ remains the associated travel distance from vertex $i$ to vertex $j$.

Problem (D): Capacitated Vehicle Routing with Time Windows

$$
\min Z=\sum_{i=0}^{n+1} \sum_{j=0}^{n+1} \sum_{k=1}^{K} c_{i j} x_{i j k} \quad i \neq j
$$

Subject to:

$$
\begin{aligned}
& \sum_{k=1}^{K} \sum_{j=1}^{n} x_{i j k}=1 \quad \forall i, \quad i \in N \\
& \sum_{j=1}^{n+1} x_{0 j}=1 \quad \forall k, k \in K \\
& \sum_{i \neq j}^{n} x_{i j k}-\sum_{i \neq j}^{n} x_{j i k}=0 \quad \forall k \in K, \forall j \in N . \\
& \sum_{i=0}^{n} x_{i j k}=1 \quad j=n+1, \quad \forall k, k \in K \\
& w_{j k} \geq w_{i k}+s_{i}+t_{i j}-M_{i j}\left(1-x_{i j k}\right) \quad \forall i, j \in V, \quad \forall k \in K \\
& w_{i k} \geq a_{i} \quad \forall i, i \in V, \quad \forall k, k \in K \\
& w_{i k} \geq b_{i}-s_{i} \quad \forall i, i \in V, \quad \forall k, k \in K \\
& \sum_{i=0}^{n+1} \sum_{j=0}^{n+1} d_{i} x_{i j k} \leq C \quad \forall k, k \in K
\end{aligned}
$$

Equation (1) is the total distance to be minimised. Equation (2) makes sure each customer is visited once. Equation (3) ensures each vehicle is used exactly once. Equation (4) is the flow conservation. Equation (5) makes sure each vehicle that departed the micro hub (index $=0$ ) returns to the micro hub (index $=n+1)$. Equation (6) ensures that time variables are consistent. The variable $M_{i j}$ is a constant, value of which is calculated as follows: $M_{i j}=\max \left\{0, b_{i}+s_{i}+t_{i j}-a_{i}\right\}$ (Cordeau et al., 2007). $M_{i j}$ is a constant to help calculate the time at which vehicle $k$ starts servicing vertex $j$ $\left(w_{j k}\right)$ considering the time needed for servicing vertex $i\left(s_{i}\right)$ and the travel time from vertex $i$ to vertex $j\left(t_{i j}\right)$. If the vehicle travels to vertex $j$ from vertex $i$, then the term including $M_{i j}$ is zero because $x_{i j k}$ will be one and the $w_{j k} \geq w_{i k}+s_{i}+t_{i j}$, ie the 
service start time of vertex $j$ is later than the service start time of vertex $i$ plus the time it takes to service vertex $i$ plus the time it takes to travel from vertex $i$ to vertex $j$. If the vehicle does not travel to vertex $j$ from vertex $i$ then the calculation of the constant $M_{i j}$ makes sure $w_{j k} \geq w_{i k}+a_{i}-b_{i}$; ie allowing $w_{j k}$ to be earlier than $w_{i k}$ by the duration of the time interval $\left[a_{i}, b_{i}\right]$ given to vertex $i$. Equations (7) and (8) impose the time windows in terms of when the vehicle can start its journey and when it finishes, which also eliminate sub-tours. Finally, Equation (9) makes sure the vehicle capacity is not exceeded.

For calculation of distances between vertex $i$ and vertex $j$, we use the latitude and the longitude of each customer to estimate the distance between the two vertices using the 'distGeo' function, which is a highly accurate estimate of the shortest distance between two points on an ellipsoid, within the 'geosphere' package in R (Hijmans, 2019). We understand using this type of a function would induce some error in the actual distances incurred; however, for the purposes of practicality and the necessity to estimate distances between tens of customers for each day throughout the analysis period of 364 days we revert to this approach which is also followed by several other researchers (Andelmin \& Bartolini, 2019; Tempelmeier et al., 2019).

We solve Problem (D), D for distribution, for each capacity, for each logistics operation (independent and shared), and for each day throughout the analysis period of 364 days. We focus on Tottenham Hale (N17 6, Figure 3) that has the highest number of orders estimated from primary data. 


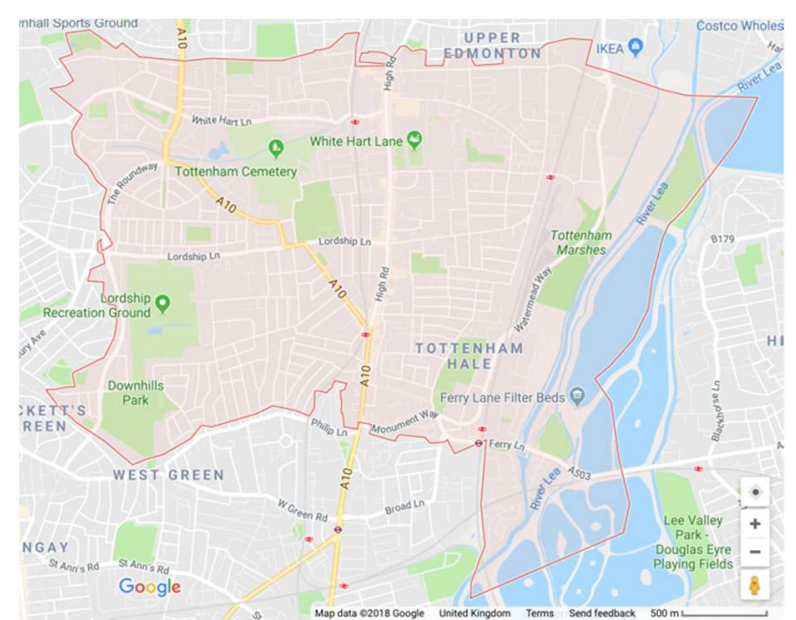

Figure 3 Tottenham Hale postcode sector

Tottenham Hale has approximately 7,600 households and a population of about 20,000 (2011 census). We assume the travel speed for small flows to be $11.91 \mathrm{kmph}$ (O'Sullivan, 2016) to calculate the $t_{i j}$ in Problem (D). Service time is assumed to be 10 minutes for parking and 2 minutes for delivering the order in line with the industry observations of the researchers, avoiding double counting of parking time when there are more than one orders from the same postcode. Vehicle capacity is assumed to be 10 orders and 15 orders in line with industry observations. In the primary data we received from the online retailer, one year was considered to be 364 days, only excluding the Christmas day when there are no deliveries. Hence, the number of VRP instances we ran is $2 * 3 * 364=2184$, where the multiplier 2 is for the two vehicle capacities: 10 and 15 orders and the multiplier 3 is for the nature of the logistics operation: two independent solutions for each of the retailers and one solution for the shared vehicle.

We illustrate the problem structure with a particular instance from our set of problem instances. Consider a distribution network that consists of one micro hub and 15 customers with their corresponding home delivery time windows. The orders have been placed by 15 customers located in 14 different postcodes (Customers 3 and 4 are in the same postcode) within the N17 6 Postcode Sector and the vehicle capacity is 10 . 
The data of this distribution network is given in Table 1. For example, Customer 3

requests its grocery delivery to be completed between 9:00 and 9:59 whereas Customer 4 requests its grocery delivery between 11:00 and 11:59.

Table 1 Latitude, Longitude, and Delivery Windows for an Illustrative Problem Instance

\begin{tabular}{|l|l|l|l|l|l|}
\hline Point & Postcode & Latitude & Longitude & $\begin{array}{l}\text { Delivery } \\
\text { Window Opens }\end{array}$ & $\begin{array}{l}\text { Delivery } \\
\text { Window Closes }\end{array}$ \\
\hline 0 & Micro Hub & 51.5933036 & -0.079255 & $05: 00$ & $05: 55$ \\
\hline 1 & N17 6AL & 51.593036 & -0.091606 & $06: 00$ & $06: 59$ \\
\hline 2 & N17 6BA & 51.591808 & -0.091845 & $06: 00$ & $06: 59$ \\
\hline 3 & N17 6BY & 51.592394 & -0.07978 & $09: 00$ & $09: 59$ \\
\hline 4 & N17 6BY & 51.592394 & -0.07978 & $11: 00$ & $11: 59$ \\
\hline 5 & N17 6DR & 51.591705 & -0.081685 & $12: 00$ & $12: 59$ \\
\hline 6 & N17 6EP & 51.595857 & -0.075461 & $14: 00$ & $14: 59$ \\
\hline 7 & N17 6EY & 51.596824 & -0.077412 & $15: 00$ & $15: 59$ \\
\hline 8 & N17 6JU & 51.591008 & -0.083043 & $16: 00$ & $16: 59$ \\
\hline 9 & N17 6LU & 51.595059 & -0.082223 & $16: 00$ & $16: 59$ \\
\hline 10 & N17 6TF & 51.594114 & -0.077209 & $18: 00$ & $18: 59$ \\
\hline 11 & N17 6TL & 51.595556 & -0.072485 & $20: 00$ & $20: 59$ \\
\hline 12 & N17 6UH & 51.593456 & -0.072184 & $20: 00$ & $20: 59$ \\
\hline 13 & N17 6UY & 51.595863 & -0.070884 & $21: 00$ & $21: 59$ \\
\hline 14 & N17 6XR & 51.591666 & -0.074367 & $22: 00$ & $22: 59$ \\
\hline 15 & N17 6YA & 51.592501 & -0.075343 & $22: 00$ & $22: 59$ \\
\hline
\end{tabular}

The optimal solution is displayed in Figure 4, the total distance is $5.27 \mathrm{~km}$ and requires two vehicles. The vehicle routes are: $0-1-2-5-8-9-0$ and $0-3-$ $4-6-7-10-12-11-13-14-15-0$. 


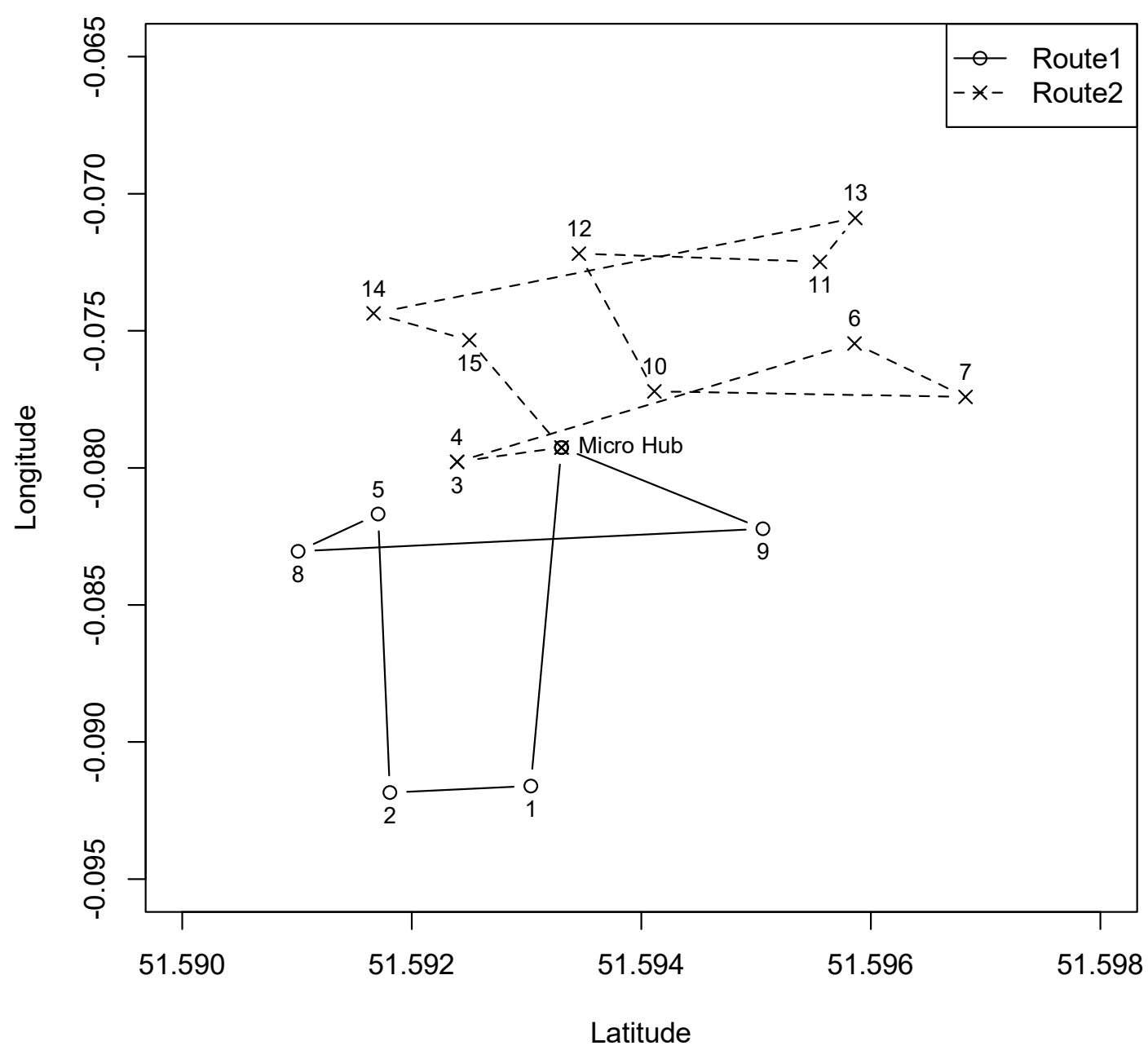

Figure 4 CVRPTW solution of the first problem instance.

It is notable that our methodology uses Monte Carlo simulation to estimate daily and hourly demand for grocery deliveries at the postcode level. We generate daily demand for a year for two retailers and solve Problem (D) instances for each retailer operating independently and collaboratively when sharing different sizes of vans.

\section{$4 \quad$ Findings}

Table 2 presents the input data for small flows from micro hubs to consumers' preferred locations over a period of 364 days. As can be seen in Table 2, the total demand of 
Retailer B is approximately 3.5 times that of Retailer A. The maximum daily demand for Retailer A is 14 and for Retailer B is 29 , whereas when the demand for both retailers are combined, the maximum daily demand is 31 , suggesting collaboration does not increase the fleet size required to meet the joint demand significantly.

Table 2 Input data (in orders) for CVRPTWs

\begin{tabular}{|l|l|l|l|l|}
\cline { 2 - 5 } \multicolumn{1}{c|}{} & $\begin{array}{l}\text { Annual } \\
\text { Demand }\end{array}$ & $\begin{array}{l}\text { Daily } \\
\text { Average }\end{array}$ & $\begin{array}{l}\text { Daily } \\
\text { Standard Deviation }\end{array}$ & $\begin{array}{l}\text { Daily } \\
\text { Maximum }\end{array}$ \\
\hline Retailer A & 1,410 & 4 & 2 & 14 \\
\hline Retailer B & 5,007 & 14 & 4 & 29 \\
\hline Joint & 6,417 & 18 & 5 & 31 \\
\hline
\end{tabular}

We solve each problem instance using AMPL with CPLEX Solver to optimality. We have two performance objectives to assess the impact of shared logistics operation under two capacities: the total distance incurred to fulfil the grocery home delivery and the number of routes. Table 3 shows the mean and the standard deviation in brackets of the total distance in $\mathrm{km}$ per day for each retailer, followed by independent operation, which is the sum of the distance to be travelled by Retailer A and Retailer B for each day, shared logistics operation, and percentage reduction from shared logistics operation.

Table 3 Mean (Standard Deviation) of total distance $(\mathrm{km})$ and distance reduction for vehicle capacity of 10 and 15 orders

\begin{tabular}{|l|l|l|}
\cline { 2 - 3 } \multicolumn{1}{c|}{} & Vehicle Capacity $=10$ orders & Vehicle Capacity =15 orders \\
\hline Retailer A & $1.404(1.061)$ & $1.404(1.061)$ \\
\hline Retailer B & $6.045(1.370)$ & $6.335(1.617)$ \\
\hline Independent Operation & $8.652(2.096)$ & $8.945(2.306)$ \\
\hline Shared Logistics & $6.929(1.367)$ & $7.120(1.612)$ \\
\hline Distance Reduction & $17 \%(28 \%)$ & $17 \%(37 \%)$ \\
\hline
\end{tabular}


Table 3, the total distance travelled is not reduced by higher capacity, but it is reduced by $17 \%$ on average by sharing vehicles. One of the reasons for the lack of distance reduction by increasing capacity for the last mile is the number of consumers to be visited per day. As is reported in Table 2, the daily average demand is usually less than 10 for Retailer A and less than 15 for Retailer B. Hence, a vehicle capacity increase does not make a difference for the independent operation. Moreover, vehicle capacity increase does not reduce the distance travelled for small flows, because the combination of customers that can be fitted in a vehicle is dependent on the time windows of their order. For example, the vehicle runs out of operating time before it runs out of capacity due to sparsity of customer orders throughout the day. On the other hand, the total distance is significantly reduced by logistics sharing as can be observed in Figure 5 for vehicle capacity equal to 10 orders and 15 orders.

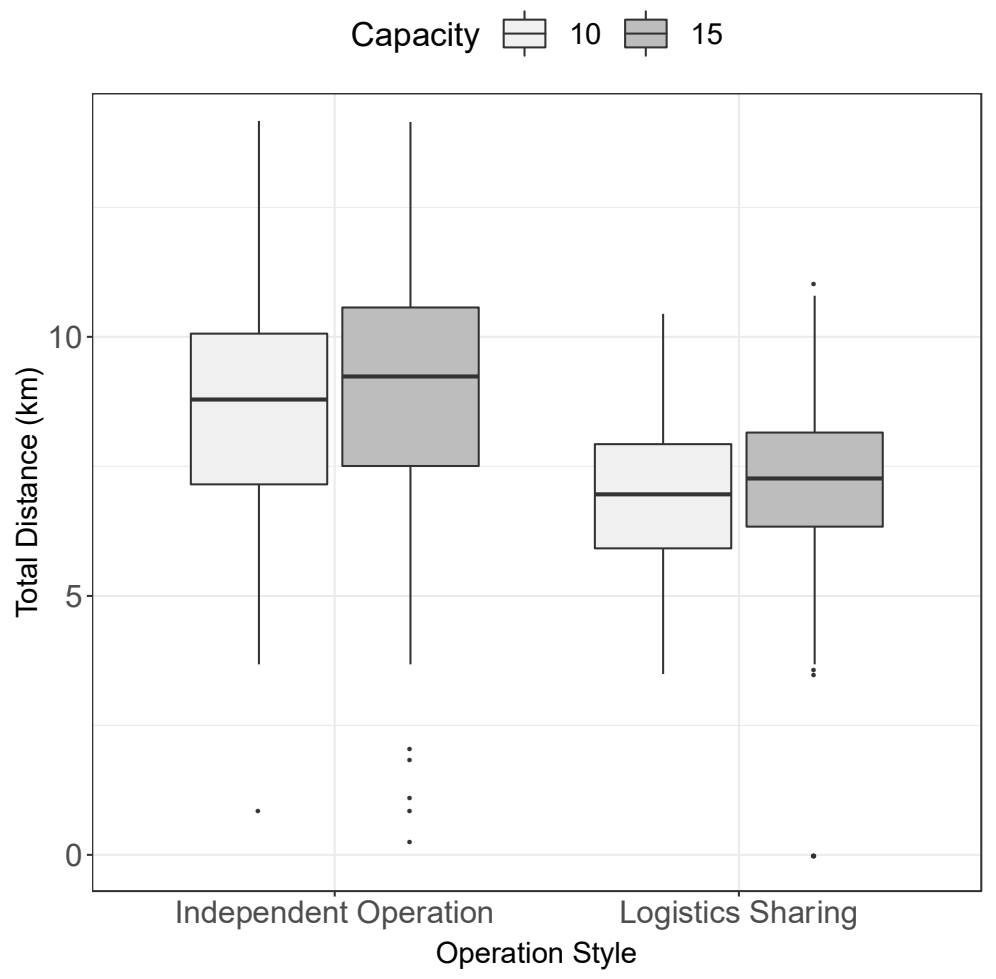

Figure 5 Total distance by vehicle capacity for both independent and shared operation 
Table 4 presents the average number of routes needed for each postcode sector for each retailer over the analysis period of 364 days and under the two operational modes: independent operation and shared logistics.

Table 4 Mean (Standard Deviation) of number of routes and route reduction for vehicle capacity of 10 and 15 orders

\begin{tabular}{|l|l|l|}
\cline { 2 - 3 } \multicolumn{1}{c|}{} & Vehicle Capacity =10 orders & Vehicle Capacity = 15 orders \\
\hline Retailer A & $0.898(0.303)$ & $0.898(0.303)$ \\
\hline Retailer B & $1.832(0.448)$ & $1.349(0.477)$ \\
\hline Independent Operation & $2.808(0.499)$ & $2.319(0.501)$ \\
\hline Shared Logistics & $2.176(0.562)$ & $1.684(0.477)$ \\
\hline Route Reduction & $22 \%(17 \%)$ & $26 \%(21 \%)$ \\
\hline
\end{tabular}

In Table 4, vehicle capacity increase does not lead to a route reduction for Retailer A, as its daily orders are fewer than or equal to 10 with some days without any grocery delivery demand. However, we observe the effect of vehicle capacity increase for Retailer B. Shared logistics operation reduces the average number of routes needed, as the grocery deliveries within the postcode sector range from 0 to 29 for individual retailers and 2 to 31 for the joint operation. Over the analysis period of 364 days, the number of routes needed to meet the grocery delivery demand is reduced by one unit for $63 \%$ of the days.

Table 5 shows the distribution of days for which 1,2,3, or 4 routes are needed to meet the demand. For example, in the analysis period, when the vehicle capacity is 10 orders, there were four days where one route was needed if the retailers operated independently and 29 days with one route under logistics sharing. 
Table 5 Days with the number of routes for vehicle capacities of 10 and 15 orders

\begin{tabular}{|l|r|r|r|r|r|}
\hline & & \multicolumn{4}{|c|}{ Operation Style } \\
\hline & \# of Routes & \multicolumn{2}{|c|}{ Independent } & \multicolumn{2}{|c|}{ Sharing } \\
\hline & & \# of Days & Percentage & \# of Days & Percentage \\
\hline \multirow{3}{*}{ Capacity =10 } & $\mathbf{1}$ & 4 & $1 \%$ & 29 & $8 \%$ \\
\cline { 2 - 7 } & $\mathbf{2}$ & 75 & $21 \%$ & 244 & $67 \%$ \\
\cline { 2 - 7 } & $\mathbf{3}$ & 272 & $75 \%$ & 89 & $24 \%$ \\
\hline \multirow{3}{*}{ Capacity $=15$} & $\mathbf{4}$ & 13 & $4 \%$ & 2 & $1 \%$ \\
\cline { 2 - 7 } & $\mathbf{1}$ & 6 & $2 \%$ & 117 & $32 \%$ \\
\cline { 2 - 7 } & $\mathbf{2}$ & 236 & $65 \%$ & 245 & $67 \%$ \\
\hline
\end{tabular}

As can be seen in Table 5, for a vehicle capacity of 10 orders, the percentage of days with three routes is reduced from $75 \%$ in the independent operation to $24 \%$ in the shared operation whereas the number of days with three routes is reduced from $34 \%$ to $1 \%$ for a vehicle capacity of 15 orders.

Whilst the distances to be covered under two vehicle capacities are similar for the independent operation (Figure 5); the number of routes for each capacity under each operation is different (Tables 4 and 5). The distance covered does not change with increasing capacity because higher capacity means fewer, longer routes.

\subsection{Comparisons of Independent and Shared Logistics}

We compare the independent operation and shared logistics on total distance and number of routes using the non-parametric Wilcoxon signed rank test instead of the paired Student's t-test, because the metrics are not normally distributed for each retailer and vehicle capacity. We report the mean rank difference in the respective metric (total distance and number of routes) followed by the probability that this difference is equal to zero in parentheses. The low probability shows that it is unlikely for the difference to be zero (ie unlikely that the two operations have no difference). Table 6 shows the 
difference between independent operation and shared logistics for distance and number of routes.

Table 6 Wilcoxon Signed Rank Test for independent and shared operation

\begin{tabular}{|l|l|l|}
\cline { 2 - 3 } \multicolumn{1}{c|}{} & Vehicle Capacity $=10$ orders & Vehicle Capacity $=15$ orders \\
\hline Distance & $1.770(0.000)$ & $1.888(0.000)$ \\
\hline Number of Routes & $0.500(0.000)$ & $0.500(0.000)$ \\
\hline
\end{tabular}

It can be concluded from Table 6 that shared logistics leads to lower distance and fewer routes for each vehicle capacity. The difference for Tottenham Hale is 1.770 $\mathrm{km}$ and $1.888 \mathrm{~km}$ for vehicle capacity of 10 and 15 orders, respectively. In terms of the number of routes; vehicle capacity does not have an impact on the level of route reduction for Tottenham Hale; the route reduction is 0.5 units for both capacities when shared logistics is implemented.

\section{Discussion}

Competitive intensity in the UK grocery retail sector has recently increased with new market entrants, such as AmazonFresh and the German discounter Aldi that launched their new online initiatives. Equally, retailers have limited opportunity to counter this lack of profitability due to the cut-throat competition while consumers are unwilling to pay for the full costs of home delivery (Asdemir et al., 2009). To address these challenges, retailers have attempted to mitigate the costs by introducing higher minimum basket spend, increasing click $\&$ collect facilities and differential delivery charges, to even out costly peak periods in demand (Zissis et al., 2017). However, these strategies alone are insufficient to reduce retailers' costs and increase their last mile delivery operational performance.

Shared logistics for last mile deliveries can be increasingly favourable when the demand density increases, since vans will be both more fuel- and time- efficient in 
delivering small loads with many stops. The shared logistics grocery distribution model presented in this paper suggests that significant benefits can be achieved through distribution efficiency gains and reduced environmental impacts. We also anticipate reduced fixed asset costs from the higher utilisation of vehicles and efficiency from micro hubs. However, in this work, we do not consider inventory benefits as our approach focuses on logistics sharing and not merging the operations entirely. On the other hand, there are obstacles to overcome in shared last mile networks, with the continued intense competitive environment, the very tight profit margins, and increasing environmental legislation; hence, we experience increased pressure on retailers to explore collaborative models as a method to achieve profitability from their home delivery operations.

Collaboration in the last mile not only has economic implications, but it also leads to environmental benefits through reduction of distance travelled. This is possible due to a strong correlation between the distance travelled and carbon emissions (Zissis, Saharidis, et al., 2018). Additionally, there may be benefits from reduced costs associated with Ultra High Emission Zones within the UK, and service benefits through the risk pooling effect of customer delivery slots leading to more choice and availability for consumers. The benefits from collaboration could be directly linked to two of the 17 Sustainable Development Goals (SDGs) adopted by the members of the United Nations (https://sustainabledevelopment.un.org/). Specifically, collaboration can help contribute to achieving SDG 11: Sustainable Cities and Communities through reduction in the number of vehicles needed for the delivery operation; hence, lower traffic congestion and noise pollution and SDG 13: Climate Action through emission reductions from shorter distances travelled and fewer vehicles needed to execute the operation.

Despite showing the opportunities to improve profitability of online grocery 
retailing, we concede that a number of commercial realities would need to be addressed. While horizontal collaboration is not a new concept (Accorsi et al., 2018) the UK grocery market is fiercely competitive and limited examples of collaboration exist. Grocery retailers currently compete in the last mile delivery; through offering shorttimed delivery windows, product availability, and differential delivery charges. Within a shared collaborative network, the ability to differentiate in the delivery service would be somewhat negated, as the operational performance would be the same for all actors utilising the network (Emeç et al., 2016). In a shared network, retailers work together by sharing resources, information and coordinating delivery routes to optimise the entire system. When retailers cooperate on distribution, they would need to re-orientate their competitive differentiation through alternative methods; pricing, offers, assortment, freshness, personalisation, etc. rather than delivery performance (Teller et al., 2016). As modelled in our simulation, we concede that it is unlikely that retailers would have equal volumes being distributed through the shared network. Retailers would, therefore, need to consider carefully the commercial arrangements of the rules for gainsharing. We envisage that, in a shared network of unequal partners, larger retailers might expect to receive a disproportionate return from the benefits and would certainly seek to ensure that smaller retailers do not receive greater benefits from gaining access to a larger network at their expense. As previously stated, coordinating a shared network necessitates the sharing of commercially sensitive data to allow for the effective planning of overall network efficiency albeit possible concerns to be raised from relevant authorities (e.g. UK Competition Commission). Processes, protocols, and systems would need to be developed that would allow effective planning while maintaining the security of data. Moreover, new real time data analytics and decision 
support systems would be needed for allocation of customers' orders for all retailers within the collaborative network.

While our model presents the operational benefits from collaborating, third party organisations could play a role in facilitating collaboration using information and communication technologies. We anticipate that the third-party logistics service providers could be the orchestrators of these shared networks, managing the coordination of integrated replenishment planning, inventory management, and the last mile physical distribution. As the concept of the sharing economy gathers pace, as exemplified by delivery models from Uberfreight (https://www.uberfreight.com) and Instacart (https://www.instacart.com), new third party integrators could provide opportunities to migrate and scale up more rapidly than the comparatively traditional incumbent service providers.

Collaboration on logistics assets can support retailers towards achieving the triple bottom line efficiencies and other business advantages. To operationalise the above, retail managers will need to be "educated" for these efficiencies, benefits, and advantages emanating from collaboration through logistics sharing. Our findings inform retailers about potential savings and efficiency increases from collaboration, paving the way for changing the mindset around logistics sharing in the last mile distribution. The competitive nature of the market puts extra pressure on retailers operating their own fleets. These results can support policies incentivising logistics sharing approaches and decoupling the last mile delivery from core grocery retail service provided by UK retailers.

Implementation of collaboration among competitors still poses several challenges especially on the extremely competitive food retail market. This could provide an excellent platform for a case study to be developed which can be used with 
both university students and executive programme participants. This case study could focus on the contemporary challenge of urbanisation and the need to consider future urban infrastructure in relation to the last mile logistics and the significant role of online retailing. This need is more intense in areas such as Asia and Africa. Herewith, different reasons prevail, especially when Asia witnessed the biggest increase in e-commerce globally in 2017 , with $44 \%$ growth while the penetration rate has reached more than $60 \%$ of its population; notably, the penetration rate both in Europe and in US is around 30\% (Kantar Worldpanel, 2017). Similarly, Africa's e-commerce sector is expected to dramatically grow over the next decade (George, 2019) with grocery delivery companies and start-ups in several countries: "supermart.ng" in Nigeria, "asbeza.net" in Ethiopia, "Trolley App" in Egypt, "Fast and Fresh" in Tanzania, and "OneCart" in South Africa.

\section{Conclusion}

This study estimated efficiency improvements from collaboration through the concept of micro hubs, which are envisaged to be placed in residential areas with a 2$\mathrm{km}$ service radius operating shared vehicles to fulfil the last mile of grocery deliveries. The gains from collaboration in the last mile delivery of grocery orders are reported as distance reduction and route reduction under two operating capacities: 10 and 15 orders per route. The distance reduction is $17 \%$ on average with a standard deviation of $28 \%$ for 10 orders per route and $37 \%$ for 15 orders per route. Route reduction is $22 \%$ and $26 \%$ on average with a standard deviation of $17 \%$ and $21 \%$ for a vehicle capacity of 10 and 15 orders per route, respectively. Our contribution is in the emerging area of vehicle sharing for the last mile delivery of grocery orders. We report the ranges for distance savings from collaboration and the circumstances where collaboration does not lead to savings: this happens when a collaborating retailer has very low demand, ie one or two 
orders per day for home deliveries. Theoretically, collaboration is expected to reduce total distance covered and we showed empirically when and by how much it reduces the total distance.

In terms of managerial implications, our work has demonstrated the concept of a micro hub operated collaboratively in residential areas with significant distance reduction in the last mile. This micro hub concept needs to be considered carefully by retail managers considering that urbanisation is a major trend that is expected to continue. Managers need to start devising alternative online retail logistics strategies where the micro hub concept will be a central theme and where many innovative features and characteristics will materialise, especially in the last mile delivery. Overall, retail managers should foster continuous innovation in their operations and employ collaborative operational approaches with their competitors. We also anticipate our work to be beneficial to various stakeholders including policymakers and local government officials considering the future environmental challenges faced by most major cities.

There are a number of limitations emanating from this work which need to be mentioned. A key limitation is the use of primary data from only one major retailer which was supported by secondary data. Our approach is scientifically robust, even though we would prefer to have primary data from at least two retailers. Another limitation is the focus on the UK online retail market which may create concerns for the wider applicability of our work, nevertheless, our results will generate many insights for online retailers with operations in other national markets considering that the UK retail sector is one of the most advanced retail sectors globally. In our experiments, we used realistic parameters such as 1-hour delivery windows, 10 minutes for parking time, and 2 minutes for delivering the order for the UK market; hence, it is necessary to adjust the 
values of these parameters before applying our model in other national markets. One of the limitations is the assumption that all consumers receive their orders within their selected time window, which may not be realistic at times due to traffic congestions or delays in previous parts of the journey.

In this work, we assumed that these micro hubs will command strong operational capabilities and will follow high quality standards in the treatment of food products; however, this assumption may prove challenging. Therefore, future work could consider how these operating standards, processes, and quality standards can be implemented accurately and homogeneously across micro hubs, generating further trust in their use in the last mile logistics. In addition, we have not considered the profit margins of individual orders that are to be delivered within the 2-km radius of the micro hubs. Such extensions could be considered in future work as the demand for online grocery purchases and the subsequent home deliveries increase. A further natural extension of our work could incorporate electric delivery vehicles. The range of electric vehicles is increasing and they will be more preferable from emissions point of view when there is no need for en-route recharging (Breunig et al., 2019). Finally, future research could explore other national, online retail markets but it could also examine the use of shared regional distribution centres and warehouses at primary distribution level. Future research could also analyse the optimal location of micro hubs serving urban environments and it could employ other methodological approaches (e.g. life cycle analysis) to investigate other environmental cost trade-offs emanating from the use of collaborative logistics by online grocery retailers.

\section{Acknowledgement}

This paper is an outcome of a project which has received funding from the European Union's Horizon 2020 research and innovation programme. 


\section{References}

Abdulkader, M., Gajpal, Y., \& ElMekkawy, T. (2018). Vehicle routing problem in omni-channel retailing distribution systems. International Journal of Production Economics, 196, 43-55. https://doi.org/10.1016/j.ijpe.2017.11.011

Accorsi, R., Baruffaldi, G., Manzini, R., \& Tufano, A. (2018). On the design of cooperative vendors' networks in retail food supply chains: A logistics-driven approach. International Journal of Logistics Research and Applications, 21(1), 35-52. https://doi.org/10.1080/13675567.2017.1354978

Al Sunny, S. M. N., Liu, X. F., \& Shahriar, M. R. (2019). An Integrated IoT Enabled On-Demand Grocery Shopping and Delivery Cloud System Using MTComm at the Edge. Proceedings - 2019 IEEE International Conference on Edge Computing, EDGE 2019 - Part of the 2019 IEEE World Congress on Services, 51-55. https://doi.org/10.1109/EDGE.2019.00024

Andelmin, J., \& Bartolini, E. (2019). A multi-start local search heuristic for the green vehicle routing problem based on a multigraph reformulation. Computers $\&$ Operations Research, 109, 43-63.

Asdemir, K., Jacob, V., \& Krishnan, R. (2009). Dynamic pricing of multiple home delivery options. European Journal of Operational Research, 196(1), 246-257. https://doi.org/10.1016/j.ejor.2008.03.005

Belavina, E., Girotra, K., \& Kabra, A. (2017). Online Grocery Retail: Revenue Models and Environmental Impact. Management Science, 63(6), 1781-1799. https://doi.org/10.1287/mnsc.2016.2430

Breunig, U., Baldacci, R., Hartl, R. F., \& Vidal, T. (2019). The electric two-echelon vehicle routing problem. Computers and Operations Research, 103, 198-210. https://doi.org/10.1016/j.cor.2018.11.005 
Cachon, G. (2014). Retail Store Density and the Cost of Greenhouse Gas Emissions. Management Science, 60(8), 1907-1925. https://doi.org/10.1287/mnsc.2013.1819

Clarke, G., Johnson, A., \& Nankivell, J. (2014). Van Travel Trends in Great Britain. https://www.racfoundation.org/wpcontent/uploads/2017/11/van_report_aecom_100414.pdf

Cleophas, C., \& Ehmke, J. F. (2014). When Are Deliveries Profitable?: Considering Order Value and Transport Capacity in Demand Fulfillment for Last-Mile Deliveries in Metropolitan Areas. Business and Information Systems Engineering, 6(3), 153-163. https://doi.org/10.1007/s12599-014-0321-9

Cordeau, J.-F., Laporte, G., Savelsbergh, M. W. P., \& Vigo, D. (2007). Chapter 6 Vehicle Routing. In C. Barnhart \& G. Laporte (Eds.), Transportation (Vol. 14, pp. 367-428). Elsevier. https://doi.org/10.1016/S0927-0507(06)14006-2

de Kervenoael, R., Yanık, S., Bozkaya, B., Palmer, M., \& Hallsworth, A. (2016). Trading-up on unmet expectations? Evaluating consumers' expectations in online premium grocery shopping logistics. International Journal of Logistics Research and Applications, 19(2), 83-104. https://doi.org/10.1080/13675567.2015.1023186

Edwards, J. B., McKinnon, A. C., \& Cullinane, S. L. (2010). Comparative analysis of the carbon footprints of conventional and online retailing. International Journal of Physical Distribution \& Logistics Management, 40(1/2), 103-123. https://doi.org/10.1108/09600031011018055

Emeç, U., Çatay, B., \& Bozkaya, B. (2016). An Adaptive Large Neighborhood Search for an E-grocery Delivery Routing Problem. Computers \& Operations Research, 69, 109-125. https://doi.org/10.1016/j.cor.2015.11.008 
George, E. (2019, June 25). US risks handing Africa's e-commerce sector to China. Financial Times. https://www.ft.com/content/d96ee9a8-9671-11e9-8cfb$30 \mathrm{c} 211 \mathrm{dcd} 229$

Hackney, R., Grant, K., \& Birtwistle, G. (2006). The UK grocery business: Towards a sustainable model for virtual markets. International Journal of Retail \& Distribution Management, 34(4/5), 354-368. https://doi.org/10.1108/09590550610660279

Hijmans, R. J. (2019). Introduction to the "geosphere" package (Version 1.5-10). https://cran.r-project.org/web/packages/geosphere/vignettes/geosphere.pdf Kantar Worldpanel. (2017). The Future of E-Commerce in FMCG (No. 4). https://www.kantarworldpanel.com/dwl.php?sn=publications\&id=1001

Kotzab, H., \& Teller, C. (2003). Value-adding partnerships and co-opetition models in the grocery industry. International Journal of Physical Distribution \& Logistics Management, 33(3), 268-281. https://doi.org/10.1108/09600030310472005

Lim, S. F. W. T., Jin, X., \& Srai, J. S. (2018). Consumer-driven e-commerce: A literature review, design framework, and research agenda on last-mile logistics models. International Journal of Physical Distribution and Logistics Management, 48(3), 308-332. https://doi.org/10.1108/IJPDLM-02-2017-0081

Lozano, S., Moreno, P., Adenso-Díaz, B., \& Algaba, E. (2013). Cooperative game theory approach to allocating benefits of horizontal cooperation. European Journal of Operational Research, 229(2), 444-452.

https://doi.org/10.1016/j.ejor.2013.02.034

Mintel. (2018). Online Grocery Retailing UK, March 2018 Executive Summary. MINTEL. https://store.mintel.com/online-grocery-retailing-uk-march-2018 
Nathanail, E., Adamos, G., \& Gogas, M. (2017). A novel approach for assessing sustainable city logistics. Transportation Research Procedia, 25, 1036-1045. https://doi.org/10.1016/j.trpro.2017.05.477

O’Sullivan, F. (2016). London's Traffic Really Is Moving More Slowly. CITYLAB.

Paul, J., Agatz, N., Spliet, R., \& Koster, R. D. (2019). Shared Capacity Routing Problem - An omni-channel retail study. European Journal of Operational Research, 273(2), 731-739. https://doi.org/10.1016/j.ejor.2018.08.027

Rogerson, S., \& Santén, V. (2017). Shippers'opportunities to increase load factor: Managing imbalances between required and available capacity. International Journal of Logistics Research and Applications, 20(6), 581-603. https://doi.org/10.1080/13675567.2017.1306612

Teller, C., Alexander, A., \& Floh, A. (2016). The impact of competition and cooperation on the performance of a retail agglomeration and its stores. Industrial Marketing Management, 52, 6-17. https://doi.org/10.1016/j.indmarman.2015.07.010

Tempelmeier, N., Dietze, S., \& Demidova, E. (2019). Crosstown traffic-supervised prediction of impact of planned special events on urban traffic. GeoInformatica, $1-32$.

UN. (2015). World Urbanization Prospects: The 2014 Revision. United Nations, Department of Economic and Social Affairs, Population Division.

Zissis, D., Aktas, E., \& Bourlakis, M. (2018). Collaboration in urban distribution of online grocery orders. The International Journal of Logistics Management, 29(4), 1196-1214. 
Zissis, D., Aktas, E., \& Bourlakis, M. (2017). A New Process Model for Urban Transport of Food in the UK. Transportation Research Procedia, 22, 588-597. https://doi.org/10.1016/j.trpro.2017.03.048

Zissis, D., Saharidis, G. K. D., Aktas, E., \& Ioannou, G. (2018). Emission reduction via supply chain coordination. Transportation Research Part D: Transport and Environment, 62. https://doi.org/10.1016/j.trd.2018.01.014 